ORIGINAL

\title{
PATRONES GEOGRÁFICOS DE LA MORTALIDAD Y DE LAS DESIGUALDADES SOCIOECONÓMICAS EN MORTALIDAD EN ESPAÑA
}

\author{
Laura Reques (1), Estrella Miqueleiz (2), Carolina Giráldez-García (3,4) Juana M. Santos $(3,4)$, \\ David Martínez $(3,4)$ y Enrique Regidor $(3,4,5)$.
}

(1) Escuela Nacional de Sanidad, Instituto de Salud Carlos III. Madrid

(2) Departamento de Sociología. Universidad Pública de Navarra. Pamplona

(3) Departmento de Medicina Preventiva y Salud Pública, Universidad Complutense de Madrid. Madrid.

(4) Instituto de Investigación Sanitaria del Hospital Clínico San Carlos (IdISSC)

(5) CIBER Epidemiología y Salud Pública (CIBERESP). Madrid. Spain.

\section{RESUMEN}

Fundamentos: Las estimaciones sobre desigualdades socioeconómicas en mortalidad a partir de registros individuales de defunciones y población son escasas y proceden únicamente de la la ciudad de Barcelona, la Comunidad de Madrid y el País Vasco. El objetivo del presente estudio fue mostrar el patrón geográfico de mortalidad en diferentes grupos socioeconómicos, así como el de las desigualdades en mortalidad en el conjunto del territorio español.

Métodos: Se realizó el seguimiento de todos los individuos mayores de 25 años del censo de población 2001 durante siete años y dos meses para conocer su estado vital (196.470.401 personas-año a riesgo y 2.379 .558 defunciones). Se calculó la tasa de mortalidad ajustada por edad en hombres y mujeres por provincia y nivel de estudios. Las desigualdades en mortalidad provinciales se estimaron mediante la razón de tasas de mortalidad en los sujetos con nivel de estudios primarios o inferiores respecto a los sujetos con estudios universitarios.

Resultados: En mujeres, las razones de tasas más bajas - entre 1,06 y 1,16- se observaron Palencia, Segovia, Guadalajara y Ávila. Las más altas -entre 1,53 y 1,75- en Málaga, Las Palmas, Ceuta, Toledo y Melilla. En hombres, las razones de tasas más bajas -entre 1,00 y 1,12- se observan en Guadalajara, Teruel, Cuenca, La Rioja y Ávila y las más altas -entre 1,47 y 1,73- en Las Palmas, Cantabria, Murcia, Melilla y Ceuta.

Conclusiones: El patrón geográfico de las tasas de mortalidad en España varía según el nivel educativo. Las desigualdades en mortalidad muestran menor magnitud en las provincias del centro peninsular.

Palabras clave: Educación. Desigualdades en salud. Mortalidad.

Correspondencia

Laura Reques

Escuela Nacional de Sanidad

Instituto de Salud Carlos III

C/ Sinesio Delgado, 4

28029 Madrid

requeslaura@hotmail.com

DOI:
}

\section{ABSTRACT \\ Geographic Patterns of Mortality and Socioeconomic Inequalities in Mortality in Spain}

Background: Estimates of socioeconomic inequalities in mortality coming from individual data sources were only available from Madrid, the Basque Country and the city of Barcelona. The aim of this study was to show the geographical pattern of mortality in different socio-economic groups, as well as that of inequalities in mortality in the whole Spanish territory.

Methods: All people aged 25 and older in the 2001 census were followed for seven years and two months to determine their vital status (196 470401 person-years at risk and 2,379,558 deaths). The socioeconomic variable was educational level. Age-adjusted mortality rate was estimated for women and men in every province and in each category of educational level. Inequalities in mortality in each province have been estimated by the ratio of mortality in subjects with primary or lower level of education compared to subjects with university education.

Results: In women, the lowest rate ratios -between 1.06 and 1.16- are observed in Palencia, Segovia, Guadalajara, Avila and Castellon and the highest -between 1.53 and 1,75- in Malaga, Las Palmas, Ceuta, Melilla and Toledo. In men, the lowest rate ratios -between 1.00 and 1.12 - are observed in Guadalajara, Teruel, Cuenca, La Rioja and Ávila and the highest -between 1.47 and 1,73- in Las Palmas, Cantabria, Murcia, Melilla and Ceuta.

Conclusions: The geographical pattern of mortality rates in Spain varies by educational level. Inequalities in mortality by education have the lowest magnitude in central peninsular provinces.

Keywords: Education. Health inequalities. Mortality. 


\section{INTRODUCCIÓN}

El patrón geográfico de mortalidad ha sido ampliamente estudiado en España. Es conocido que tanto en hombres como en mujeres las mayores tasas se observan en el sur (Andalucía), en las islas Canarias y en algunas regiones de levante (Murcia, Comunidad Valenciana), del oeste (Extremadura) y del norte (Galicia) ${ }^{1-3}$. Sin embargo, se desconoce cuál es el patrón geográfico de la mortalidad en los distintos grupos socioeconómicos así como el de las desigualdades socioeconómicas en mortalidad.

Las estimaciones sobre desigualdades socioeconómicas en mortalidad a partir de los registros individuales de defunciones y población son escasas y proceden, únicamente, de la ciudad de Barcelona y de dos comunidades autónomas, Madrid y País Vasco $0^{4,5}$. Los estudios comparativos internacionales, en los que se han incluido estas estimaciones muestran menores desigualdades en mortalidad en España que en otros países europeos ${ }^{5,6}$. Dado que se trata de tres ámbitos geográficos con un nivel socioeconómico superior al del resto del país, es probable que esos hallazgos no sean extrapolables a otras áreas.

Otros estudios evaluaron las diferencias en mortalidad en 11 ciudades (Alicante, Barcelona, Bilbao, Castellón, Córdoba, Madrid, Málaga, Sevilla, Valencia, Vigo y Zaragoza) según el contexto socioeconómico del área de residencia, tomando la sección censal como unidad de análisis ${ }^{7,8}$. Sus hallazgos reflejan gran heterogeneidad en la magnitud de las desigualdades en mortalidad a nivel urbano. Por ejemplo, la razón de tasas que compara la mortalidad en las áreas urbanas con mayor privación material con la mortalidad en las áreas urbanas con menor privación material oscila entre 0,80 en Vigo y 1,33 en Valencia en mujeres y entre 0,97 en Vigo y 1,84 en Sevilla en hombres? ${ }^{7}$. Además, no se ha observado un patrón geográfico claro cuando se trata de la magnitud de las desigualdades en mortalidad en las ciudades estudiadas.
El objetivo del presente estudio fue mostrar el patrón geográfico de mortalidad en diferentes grupos socioeconómicos y el patrón geográfico de las desigualdades en mortalidad en España.

\section{SUJETOS Y MÉTODOS}

Se estimaron las tasas de mortalidad en sujetos con diferentes categorías de nivel de estudios y las diferencias en mortalidad según el nivel de estudios en cada una de las provincias españolas.

Se utilizó como fuente de datos la población incluida en el censo de 2001, de cuyo estado vital se realizó un seguimiento durante siete años y dos meses. Los datos fueron elaborados por el Instituto Nacional de Estadística en base a los registros individuales del censo, los cuales se cruzaron con el registro de población y con el registro de mortalidad mediante identificadores comunes. Los fallecimientos se contabilizaron entre el 1 de noviembre de 2001, fecha de realización del censo, y el 31 de diciembre de 2008, fecha de finalización del periodo de seguimiento. El Instituto Nacional de Estadística proporcionó los datos a los investigadores tras eliminar la información de carácter personal con el fin de mantener el anonimato y la confidencialidad.

La cohorte final de seguimiento quedó constituida por 40.148 .305 personas, después de excluir al 1,7\% de los sujetos por no poder localizarlos en el registro de población ni en el registro de mortalidad durante el seguimiento. Para la realización de este trabajo se seleccionó a los 28.944.854 individuos mayores de 25 años, ya que esta era la edad que les permitiría haber alcanzado el máximo nivel de estudios. En el $0,8 \%$ de los sujetos la información sobre nivel de estudios estaba ausente por lo que fueron excluidos del análisis. Finalmente se analizó a 196.470.401 personas-año en riesgo y 2.379 .558 defunciones. 
El nivel de estudios que se recogió en el censo se refería al nivel más alto completado. En base a lo expuesto, los sujetos fueron agrupados en cuatro categorías: estudios primarios o inferiores, estudios secundarios de primer ciclo, estudios secundarios de segundo ciclo y estudios universitarios. En cada provincia y en cada categoría de nivel de estudios se calcularon las tasas de mortalidad por 1.000 habitantes en mujeres y en hombres, ajustadas por edad, tomando como referencia la población estándar europea.

Las tasas de mortalidad en los sujetos con menor y mayor nivel de estudios además se representaron geográficamente mediante el índice de Jenks ${ }^{9,10}$, también llamado de "puntos de ruptura naturales". Este índice minimiza la varianza intraclase y maximiza la varianza interclase entre las categorías de distribución, por lo que la distribución de los intervalos de cada categoría se parece lo más posible a la de los clústeres naturales de la muestra. Asimismo, en cada provincia se calculó la razón de tasas de mortalidad para hombres y mujeres en los sujetos con estudios primarios o inferiores con respecto a los sujetos con estudios universitarios. Los intervalos de confianza se calcularon a partir de la varianza estimada por el método de Mantel-Haenszel para datos persona-tiempo ${ }^{11}$. Finalmente, se calcularon los coeficientes de correlación entre las tasas de mortalidad estandarizadas y la renta per cápita de cada provincia en el año 2001, para hombres y mujeres en cada uno de los grupos de nivel de estudios El análisis fue realizado utilizando el paquete estadístico spss v.21 y el programa de gestión de hojas de cálculo Excel 2010.

\section{RESULTADOS}

La tabla 1 muestra el número de personasaño en riesgo, el número de defunciones y las tasas de mortalidad general para cada una de las 50 provincias y las dos ciudades autónomas. Las tasas de mortalidad por 1.000 personas-año variaron entre 7,1 en Soria y 11,7 en Ceuta en mujeres y entre 12,1 en Guadalajara y 18,4 en Melilla en hombres.
Las tasas de mortalidad provinciales según el nivel de estudios en mujeres y en hombres se muestran en la tabla 2. Exceptuando algunas provincias donde la tasa de mortalidad es mayor en los sujetos con estudios universitarios que en los que no tenían más que bachillerato, como Ávila y Málaga tanto en hombres como en mujeres, en líneas generales en todas las provincias se observa un gradiente socioeconómico inverso, ya que los individuos con el menor y el mayor nivel de estudios presentan las tasas de mortalidad más alta y más baja, respectivamente.

En mujeres con estudios primarios o inferiores, las tasas de mortalidad por 1.000 personas-año oscilaron entre 7,3 en Soria y 12,3 en Ceuta. Otras provincias con tasas bajas y altas fueron, respectivamente, Burgos, Salamanca, Segovia, Valladolid, Guadalajara, La Rioja, Álava, Zamora, Palencia, Ávila, Madrid y Navarra, cuya magnitud fue inferior a 8 por 1.000 personas-año y Melilla, Cádiz, Sevilla, Huelva y Málaga, cuya magnitud fue superior a 10 por 1.000 personas-año. En mujeres con estudios universitarios, la tasa de mortalidad por 1.000 personas-año osciló entre 5 en Toledo y 8,6 en Huelva. Otras provincias con tasas bajas y altas de mortalidad en esta categoría de nivel de estudios fueron, respectivamente, Soria, Cuenca, Lugo, Tarragona y Zamora, cuya magnitud fue inferior a 6 por 1.000 personas-año y Castellón, Jaén, Sevilla, Ceuta y Cádiz, cuya magnitud fue superior a 7,5 por 1.000 personas-año.

En hombres con estudios primarios o inferiores, las tasas de mortalidad por 1.000 personas-año oscilaron entre 12,8 en Soria y 20,9 en Ceuta. Otras provincias con tasa bajas fueron, Ávila, Zamora, Salamanca, Segovia, Guadalajara y Cuenca, cuya magnitud resultó inferior a 13,5 por 1.000 personas-año, mientras que Melilla, Sevilla y Cádiz presentaron tasas de mortalidad por encima de 18 defunciones por 1.000 personas-año. En hombres con estudios 


\begin{tabular}{|c|c|c|c|c|c|c|}
\hline \multicolumn{7}{|c|}{$\begin{array}{l}\text { Tabla } 1 \\
\text { Personas-año a riesgo, número de defunciones y tasas de mortalidad general estandarizadas } \\
\text { por } 1.000 \text { personas-año. Cohorte de hombres y mujeres de } 25 \text { años de edad y mayores. España 2001-2008 }\end{array}$} \\
\hline \multirow[b]{2}{*}{ Provincia } & \multicolumn{3}{|c|}{ Mujeres } & \multicolumn{3}{|c|}{ Hombres } \\
\hline & $\begin{array}{c}\text { Personas/año } \\
\text { en riesgo }\end{array}$ & $\begin{array}{c}\text { Número } \\
\text { de defunciones } \\
\end{array}$ & $\begin{array}{c}\text { Tasa de } \\
\text { mortalidad general }\end{array}$ & $\begin{array}{l}\text { Personas/ año } \\
\text { en riesgo }\end{array}$ & $\begin{array}{c}\text { Número } \\
\text { de defunciones }\end{array}$ & $\begin{array}{c}\text { Tasa de } \\
\text { mortalidad general } \\
\end{array}$ \\
\hline Total & 102.054 .607 & 1.119 .532 & 8,5 & 94.400 .680 & 1.259 .898 & 14,7 \\
\hline Álava & 743.701 & 6.681 & 7,6 & 712.748 & 8.331 & 13,8 \\
\hline Albacete & 873.098 & 9.890 & 8,4 & 831.290 & 11.355 & 13,5 \\
\hline Alicante & 3.613 .450 & 36.588 & 8,5 & 3.392 .217 & 42.891 & 14,1 \\
\hline Almería & 1.207 .476 & 12.317 & 9.6 & 1.164 .193 & 14.774 & 15,9 \\
\hline Avila & 414.705 & 5.831 & 7,8 & 413.066 & 7.066 & 13,4 \\
\hline Badajoz & 1.548 .307 & 19.794 & 9,4 & 1.463 .034 & 22.107 & 16,0 \\
\hline Baleares & 2.063 .379 & 21.472 & 8,7 & 1.970 .125 & 24.411 & 14,8 \\
\hline Barcelona & 12.252 .946 & 132.407 & 8,3 & 11.073 .982 & 144.682 & 14,7 \\
\hline Burgos & 879.980 & 10.056 & 7,2 & 869.019 & 12.520 & 13,5 \\
\hline Cáceres & 900.477 & 13.972 & 8,4 & 876.523 & 13.997 & 14,7 \\
\hline Cádiz & 2.584 .315 & 25.135 & 10,2 & 2.428 .472 & 29.831 & 17,1 \\
\hline Castellón & 12.17 .911 & 14.769 & 9,1 & 1.169 .401 & 16.596 & 14,9 \\
\hline Ciudad Real & 1.163 .618 & 15.142 & 9,1 & 1.074 .987 & 16.305 & 14,3 \\
\hline Córdoba & 1.829 .141 & 21.438 & 9,1 & 1.667 .357 & 23.923 & 15,5 \\
\hline Coruña (La) & 2.945 .469 & 36.474 & 8,3 & 2.576 .978 & 38.354 & 14,7 \\
\hline Cuenca & 494.448 & 7.108 & 8,2 & 481.809 & 8.087 & 13,0 \\
\hline Girona & 1.463 .624 & 16.150 & 8,2 & 1.407 .160 & 18.693 & 13,9 \\
\hline Granada & 1.938 .730 & 21.989 & 9,6 & 1.777 .138 & 25.202 & 15,7 \\
\hline Guadalajara & 467.013 & 5.001 & 7,6 & 479.428 & 5.962 & 12,1 \\
\hline Guipúzcoa & 1.760 .596 & 18.288 & 7,9 & 1.635 .163 & 21.211 & 14,9 \\
\hline Huelva & 1.082 .930 & 13.151 & 10,1 & 1.022 .253 & 14.436 & 16,8 \\
\hline Huesca & 521.152 & 6.968 & 7,7 & 519.950 & 8.526 & 13,6 \\
\hline Jaén & 1.496 .307 & 18.282 & 9,6 & 1.394 .819 & 21.130 & 15,6 \\
\hline León & 1.282 .591 & 16.939 & 7,8 & 1.177 .587 & 19.575 & 13,7 \\
\hline Lleida & 917.766 & 12.225 & 8,6 & 890.127 & 14.079 & 14,1 \\
\hline Rioja (La) & 699.055 & 7.603 & 7,5 & 676.321 & 9.219 & 13,6 \\
\hline Lugo & 966.327 & 15.077 & 8,0 & 880.613 & 17.032 & 14,1 \\
\hline Madrid & 13.774 .346 & 121.750 & 7,6 & 12.303 .013 & 131.785 & 13,6 \\
\hline Málaga & 3.111 .536 & 31.391 & 9,1 & 2.864 .819 & 35.945 & 15,4 \\
\hline Murcia & 2.789 .655 & 29.285 & 9,5 & 2.641 .146 & 32.833 & 15,2 \\
\hline Navarra & 1.397 .741 & 14.795 & 7,6 & 1344.391 & 17.216 & 13,7 \\
\hline Orense & 918.996 & 14.229 & 7,8 & 808.615 & 15.353 & 13,9 \\
\hline Asturias & 2.906 .350 & 38.305 & 8,4 & 2.551 .624 & 42.244 & 15,7 \\
\hline Palencia & 435.302 & 5.508 & 7,8 & 416.390 & 6.572 & 14,7 \\
\hline Palmas (Las) & 2.058 .991 & 16.525 & 7,8 & 2.017 .784 & 20.889 & 15,4 \\
\hline Pontevedra & 2.326 .048 & 26.534 & 8,2 & 2.033 .030 & 27.527 & 14,8 \\
\hline Salamanca & 893.923 & 11.195 & 7,4 & 817.035 & 12.351 & 12,4 \\
\hline S. C. de Tenerife & 1.945 .557 & 17.134 & 8,7 & 1.834 .156 & 20.662 & 14,7 \\
\hline Cantabria & 1.415 .353 & 15.887 & 7,8 & 1.294 .015 & 18.377 & 14,7 \\
\hline Segovia & 367.073 & 4.655 & 7,4 & 361.330 & 5.557 & 13,0 \\
\hline Sevilla & 4.137 .523 & 44.546 & 10,0 & 3.778 .895 & 49.282 & 17,1 \\
\hline Soria & 227.250 & 3.173 & 7,1 & 228.369 & 3.732 & 12,3 \\
\hline Tarragona & 1.593 .521 & 18.034 & 8,7 & 1.542 .871 & 21.040 & 14,3 \\
\hline Teruel & 336.475 & 5.056 & 8,2 & 341.053 & 5.943 & 13,3 \\
\hline Toledo & 1.370 .577 & 15.525 & 8,2 & 1.341 .540 & 18.076 & 13,3 \\
\hline Valencia & 5.607 .446 & 63.924 & 9,4 & 5.137 .860 & 70.710 & 15,9 \\
\hline Valladolid & 1.102 .455 & 12.045 & 7,7 & 1.191 .022 & 14.134 & 13,7 \\
\hline Vizcaya & 2.984 .628 & 31.941 & 8,2 & 2.695 .717 & 37.368 & 15,3 \\
\hline Zamora & 517.551 & 7.853 & 7,5 & 493.547 & 8.682 & 12,5 \\
\hline Zaragoza & 2.234 .016 & 26.629 & 8,3 & 2.071 .762 & 30.160 & 14,4 \\
\hline Ceuta & 145.297 & 1.523 & 11,7 & 143.401 & 1.755 & 17,8 \\
\hline Melilla & 128.485 & 1.343 & 11,2 & 121.535 & 1.410 & 18,4 \\
\hline
\end{tabular}




\begin{tabular}{|c|c|c|c|c|c|c|c|c|}
\hline \multicolumn{9}{|c|}{$\begin{array}{c}\text { Tabla } 2 \\
\text { Tasas de mortalidad estandarizadas por } 1.000 \text { personas-año, por nivel de estudios en mujeres y hombres. } \\
\text { Cohorte de } 25 \text { años de edad y mayores. España 2001-2008 }\end{array}$} \\
\hline \multirow[b]{2}{*}{ Provincia } & \multicolumn{4}{|c|}{ Mujeres } & \multicolumn{4}{|c|}{ Hombres } \\
\hline & $\begin{array}{l}\text { Educación } \\
\text { primaria } \\
\text { o menos }\end{array}$ & $\begin{array}{l}\text { Educación } \\
\text { secundaria } \\
\text { de } 1^{\circ} \text { ciclo }\end{array}$ & $\begin{array}{l}\text { Educación } \\
\text { secundaria } \\
\text { de } 2^{\circ} \text { ciclo }\end{array}$ & $\begin{array}{c}\text { Estudios } \\
\text { universitarios }\end{array}$ & $\begin{array}{c}\text { Educación } \\
\text { primaria } \\
\text { o menos }\end{array}$ & $\begin{array}{l}\text { Educación } \\
\text { secundaria } \\
\text { de } 1^{\circ} \text { ciclo }\end{array}$ & $\begin{array}{l}\text { Educación } \\
\text { secundaria } \\
\text { de } 2^{\circ} \text { ciclo }\end{array}$ & $\begin{array}{c}\text { Estudios } \\
\text { universitarios }\end{array}$ \\
\hline Alava & 7,8 & 6,6 & 6,7 & 6,4 & 15,1 & 12,9 & 12,0 & 11,8 \\
\hline Albacete & 8,6 & 7,2 & 7,0 & 6,5 & 13,9 & 12,1 & 12,8 & 12,0 \\
\hline Alicante & 8,7 & 6,6 & 6,3 & 6,6 & 15,3 & 11,8 & 11,4 & 10,8 \\
\hline Almería & 9,8 & 7,7 & 8,3 & 7,2 & 16,8 & 14,0 & 14,2 & 12,1 \\
\hline Avila & 7,9 & 6,5 & 6,0 & 7,1 & 13,4 & 12,9 & 11,8 & 12,0 \\
\hline Badajoz & 9,6 & 7,0 & 7,0 & 6,9 & 16,5 & 14,7 & 13,6 & 12,3 \\
\hline Baleares & 9,0 & 7,9 & 7,1 & 6,6 & 15,9 & 14,2 & 12,8 & 11,6 \\
\hline Barcelona & 8,5 & 7,3 & 7,0 & 6,4 & 16,0 & 13,8 & 13,0 & 11,2 \\
\hline Burgos & 7,4 & 6,5 & 6,4 & 6,2 & 14,3 & 12,9 & 11,1 & 11,6 \\
\hline Cáceres & 8,5 & 6,9 & 6,7 & 6,6 & 15,0 & 13,1 & 13,9 & 11,8 \\
\hline Cádiz & 10,4 & 8,3 & 8,2 & 7,7 & 18,1 & 15,4 & 13,9 & 13,8 \\
\hline Castellón & 9,3 & 7,8 & 7,7 & 8,0 & 15,5 & 12,9 & 14,0 & 12,5 \\
\hline Ciudad Real & 9,2 & 7,1 & 7,1 & 7,1 & 14,9 & 12,6 & 13,0 & 13,1 \\
\hline Córdoba & 9,1 & 8,1 & 7,3 & 6,6 & 16,2 & 14,7 & 13,9 & 12,7 \\
\hline A Coruña & 8,6 & 8,1 & 7,4 & 7,1 & 16,0 & 13,8 & 12,9 & 11,7 \\
\hline Cuenca & 8,3 & 7,1 & 7,1 & 5,6 & 13,4 & 11,0 & 11,5 & 12,2 \\
\hline Girona & 8,3 & 7,2 & 7,3 & 6,2 & 14,9 & 12,9 & 12,7 & 10,9 \\
\hline Granada & 10,0 & 7,9 & 7,7 & 7,2 & 16,9 & 14,2 & 13,4 & 11,9 \\
\hline Guadalajara & 7,6 & 6,4 & 8,0 & 7,1 & 12,8 & 12,1 & 12,1 & 12,7 \\
\hline Guipúzcoa & 8,1 & 7,0 & 6,7 & 6,2 & 16,0 & 14,5 & 13,5 & 11,8 \\
\hline Huelva & 10,4 & 8,9 & 8,4 & 8,5 & 17,9 & 15,3 & 14,4 & 14,0 \\
\hline Huesca & 8,1 & 6,8 & 7,0 & 6,1 & 13,7 & 12,6 & 11,3 & 12,1 \\
\hline Jaén & 9,6 & 8,4 & 7,2 & 7,8 & 16,2 & 14,4 & 14,8 & 12,8 \\
\hline León & 8,0 & 6,7 & 6,6 & 6,2 & 14,8 & 12,7 & 12,1 & 11,2 \\
\hline Lleida & 9,0 & 7,5 & 6,7 & 6,7 & 15,0 & 13,3 & 12,5 & 11,0 \\
\hline Rioja (La) & 7,7 & 6,8 & 6,7 & 6,3 & 14,1 & 12,3 & 13,3 & 12,6 \\
\hline Lugo & 8,2 & 6,6 & 6,5 & 5,9 & 15,1 & 12,8 & 12,5 & 11,0 \\
\hline Madrid & 7,9 & 7,0 & 6,8 & 6,4 & 14,9 & 13,4 & 12,8 & 11,0 \\
\hline Málaga & 10,2 & 7,5 & 6,5 & 6,7 & 14,9 & 12,6 & 13,0 & 13,1 \\
\hline Murcia & 9,8 & 8,0 & 7,4 & 6,9 & 17,2 & 13,1 & 11,5 & 10,9 \\
\hline Navarra & 7,9 & 6,7 & 6,5 & 6,3 & 16,2 & 13,9 & 13,3 & 11,7 \\
\hline Orense & 8,0 & 6,3 & 6,8 & 6,2 & 14,6 & 13,2 & 13,1 & 10,7 \\
\hline Asturias & 8,7 & 7,6 & 6,8 & 6,8 & 17,1 & 15,0 & 14,4 & 12,4 \\
\hline Palencia & 7,9 & 7,7 & 7,5 & 7,4 & 15,0 & 13,9 & 12,7 & 12,5 \\
\hline Palmas (Las) & 9,8 & 7,7 & 7,9 & 6,4 & 16,6 & 13,6 & 14,6 & 11,4 \\
\hline Pontevedra & 8,5 & 6,9 & 6,6 & 6,4 & 16,0 & 12,8 & 12,7 & 11,3 \\
\hline Salamanca & 7,5 & 6,9 & 6,3 & 6,6 & 13,1 & 11,7 & 11,7 & 11,2 \\
\hline S. C. de Tenerife & 9,0 & 7,4 & 6,5 & 6,9 & 15,8 & 12,8 & 11,4 & 11,2 \\
\hline Cantabria & 8,1 & 7,0 & 7,2 & 6,6 & 16,2 & 14,3 & 13,1 & 10,9 \\
\hline Segovia & 7,6 & 7,1 & 6,5 & 7,1 & 13,4 & 12,0 & 12,0 & 11,7 \\
\hline Sevilla & 10,4 & 8,3 & 7,5 & 7,7 & 18,4 & 15,8 & 15,2 & 13,3 \\
\hline Soria & 7,3 & 6,2 & 5,9 & 5,5 & 12,8 & 10,9 & 11,6 & 10,5 \\
\hline Tarragona & 9,0 & 7,7 & 7,8 & 5,9 & 15,2 & 13,7 & 13,3 & 10,6 \\
\hline Teruel & 8,3 & 7,0 & 7,3 & 6,7 & 13,5 & 12,7 & 13,1 & 12,8 \\
\hline Toledo & 8,2 & 6,7 & 6,8 & 5,0 & 13,6 & 12,5 & 13,1 & 11,1 \\
\hline Valencia & 9,7 & 8,0 & 7,7 & 7,1 & 16,9 & 15,0 & 14,3 & 13,1 \\
\hline Valladolid & 7,6 & 6,6 & 7,0 & 6,4 & 14,6 & 12,6 & 13,4 & 12,4 \\
\hline Vizcaya & 8,6 & 7,6 & 7,4 & 7,0 & 16,8 & 15,2 & 14,2 & 12,8 \\
\hline Zamora & 7,8 & 6,6 & 6,5 & 6,0 & 12,9 & 11,4 & 13,0 & 10,7 \\
\hline Zaragoza & 8,6 & 7,5 & 7,2 & 7,3 & 15,4 & 13,6 & 14,0 & 12,2 \\
\hline Ceuta & 12,3 & 9,8 & 9,1 & 7,7 & 20,9 & 15,0 & 14,4 & 12,1 \\
\hline Melilla & 11,7 & 9,3 & 7,1 & 6,7 & 19,9 & 16,9 & 16,1 & 12,2 \\
\hline
\end{tabular}


Figura 1

Tasas de mortalidad ajustadas por edad por 1.000 personas año en riesgo y por provincia en sujetos con estudios primarios o inferiores. España, 2001-2008. A: Mujeres. B: Hombres
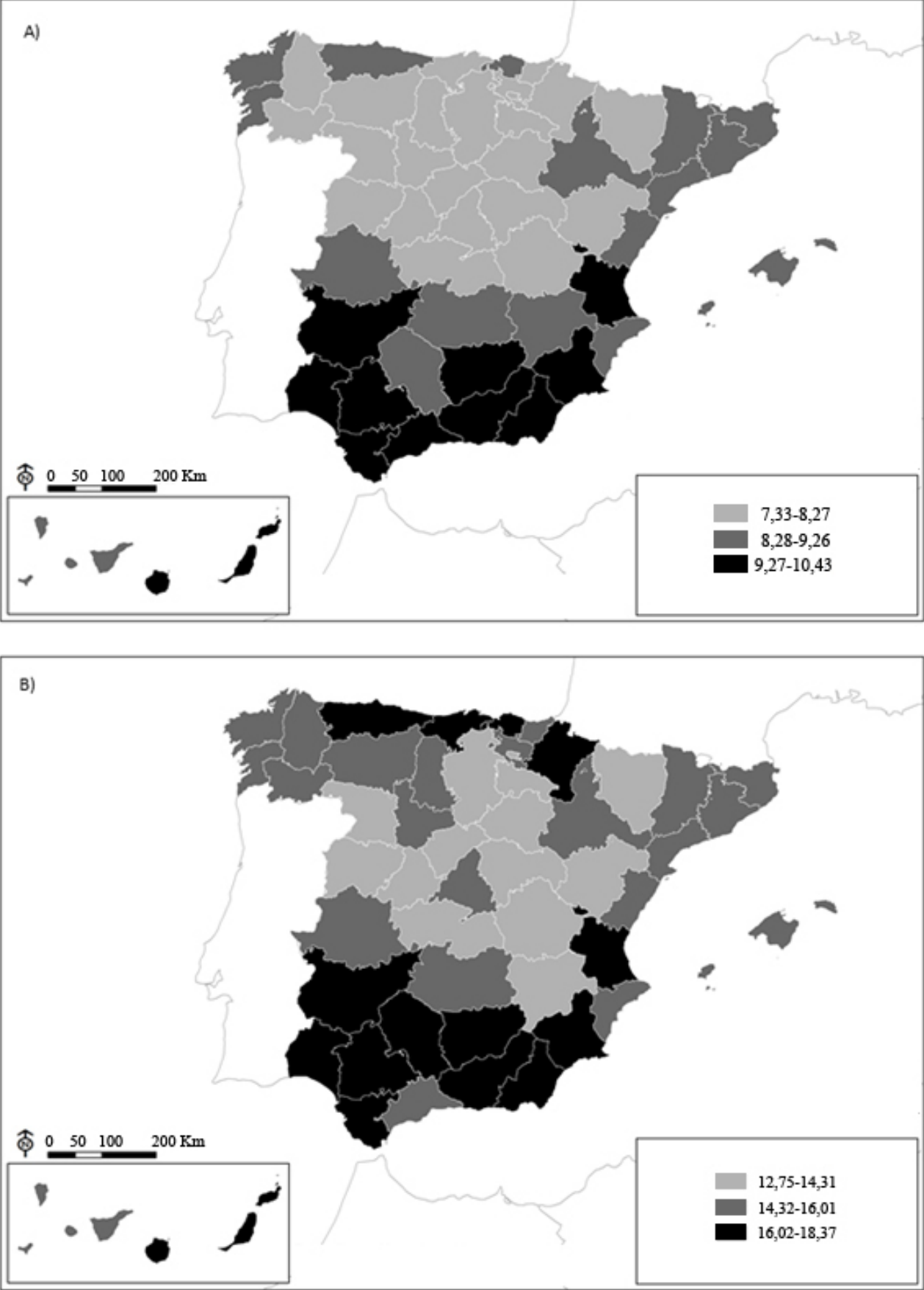
Figura 2

Tasas de mortalidad ajustadas por edad por 1.000 personas año en riesgo y por provincia en sujetos con estudios universitarios. España, 2001-2008. A: Mujeres. B: Hombres
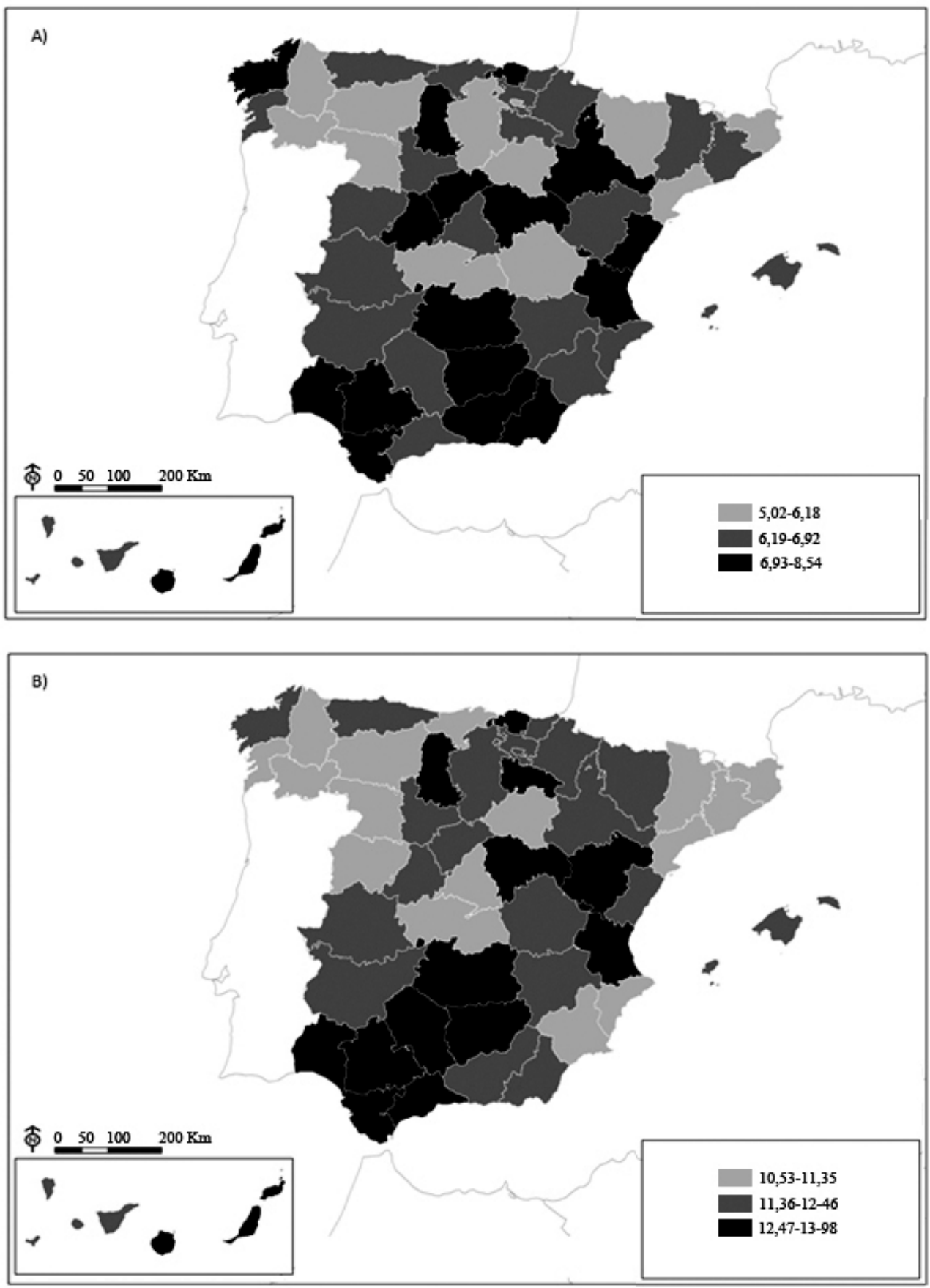
Tabla 3

Razones de tasas de mortalidad con intervalo de confianza del $95 \%$ en mujeres y hombres. Cohorte de 25 años de edad y mayores. España 2001-2008

\begin{tabular}{|c|c|c|c|c|}
\hline \multirow{2}{*}{ Provincia } & \multicolumn{2}{|c|}{ Mujeres } & \multicolumn{2}{|c|}{ Hombres } \\
\hline & $\mathrm{RT}^{*}$ & IC 95\% & $\mathrm{RT}^{*}$ & IC $95 \%$ \\
\hline Álava & 1,22 & $1,06-1,39$ & 1,27 & $1,16-1,39$ \\
\hline Albacete & 1,31 & $1,13-1,53$ & 1,16 & $1,05-1,28$ \\
\hline Alicante & 1,31 & $1,22-1,40$ & 1,42 & $1,36-1,48$ \\
\hline Almería & 1,36 & $1,21-1,54$ & 1,39 & $1,28-1,50$ \\
\hline Avila & 1,10 & $0,94-1,29$ & 1,12 & $1,02-1,32$ \\
\hline Badajoz & 1,40 & $1,25-1,56$ & 1,34 & $1,03-1,23$ \\
\hline Baleares & 1,37 & $1,26-1,51$ & 1,37 & $1,21-1,45$ \\
\hline Barcelona & 1,34 & $1,29-1,38$ & 1,42 & $1,39-1,45$ \\
\hline Burgos & 1,20 & $1,05-1,35$ & 1,23 & $1,14-1,34$ \\
\hline Cáceres & 1,30 & $1,14-1,48$ & 1,27 & $1,17-1,39$ \\
\hline Cádiz & 1,36 & $1,25-1,49$ & 1,31 & $1,25-1,38$ \\
\hline Castellón & 1,16 & $1,03-1,32$ & 1,25 & $1,15-1,35$ \\
\hline Ciudad Real & 1,29 & $1,13-1,48$ & 1,13 & $1,04-1,23$ \\
\hline Córdoba & 1,39 & $1,25-1,53$ & 1,27 & $1,20-1,35$ \\
\hline A Coruña & 1,20 & $1,13-1,28$ & 1,36 & $1,30-1,42$ \\
\hline Cuenca & 1,48 & $1,20-1,82$ & 1,10 & $0,96-1,26$ \\
\hline Girona & 1,35 & $1,21-1,51$ & 1,36 & $1,27-1,46$ \\
\hline Granada & 1,38 & $1,27-1,49$ & 1,42 & $1,35-1,50$ \\
\hline Guadalajara & 1,08 & $0,90-1,30$ & 1,00 & $0,89-1,14$ \\
\hline Guipúzcoa & 1,29 & $1,19-1,41$ & 1,35 & $1,28-1,43$ \\
\hline Huelva & 1,21 & $1,06-1,39$ & 1,28 & $1,17-1,39$ \\
\hline Huesca & 1,31 & $1,12-1,54$ & 1,13 & $1,01-1,26$ \\
\hline Jaén & 1,25 & $1,11-1,39$ & 1,27 & $1,18-1,36$ \\
\hline León & 1,31 & $1,20-1,43$ & 1,32 & $1,24-1,41$ \\
\hline Lleida & 1,32 & $1,16-1,51$ & 1,36 & $1,24-1,48$ \\
\hline Rioja (La) & 1,22 & $1,06-1,39$ & 1,12 & $1,02-1,23$ \\
\hline Lugo & 1,38 & $1,23-1,56$ & 1,37 & $1,25-1,51$ \\
\hline Madrid & 1,23 & $1,20-1,26$ & 1,36 & $1,33-1,38$ \\
\hline Málaga & 1,53 & $1,43-1,63$ & 1,13 & $1,04-1,23$ \\
\hline Murcia & 1,41 & $1,30-1,53$ & 1,58 & $1,51-1,65$ \\
\hline Navarra & 1,25 & $1,14-1,36$ & 1,39 & $1,32-1,46$ \\
\hline Orense & 1,29 & $1,14-1,46$ & 1,37 & $1,28-1,45$ \\
\hline Asturias & 1,29 & $1,21-1,37$ & 1,38 & $1,33-1,44$ \\
\hline Palencia & 1,06 & $0,91-1,24$ & 1,19 & $1,06-1,34$ \\
\hline Palmas (Las) & 1,54 & $1,40-1,69$ & 1,47 & $1,38-1,55$ \\
\hline Pontevedra & 1,33 & $1,23-1,45$ & 1,41 & $1,33-1,50$ \\
\hline Salamanca & 1,17 & $1,06-1,29$ & 1,17 & $1,09-1,25$ \\
\hline S C de Tenerife & 1,31 & $1,21-1,43$ & 1,42 & $1,34-1,50$ \\
\hline Cantabria & 1,22 & $1,12-1,34$ & 1,48 & $1,39-1,57$ \\
\hline Segovia & 1,07 & $0,91-1,29$ & 1,14 & $1,02-1,29$ \\
\hline Sevilla & 1,34 & $1,27-1,42$ & 1,38 & $1,34-1,43$ \\
\hline Soria & 1,33 & $1,09-1,65$ & 1,21 & $1,02-1,42$ \\
\hline Tarragona & 1,51 & $1,36-1,68$ & 1,43 & $1,34-1,53$ \\
\hline Teruel & 1,22 & $0,94-1,56$ & 1,06 & $0,90-1,24$ \\
\hline Toledo & 1,63 & $1,40-1,90$ & 1,23 & $1,12-1,34$ \\
\hline Valencia & 1,36 & $1,30-1,44$ & 1,29 & $1,25-1,32$ \\
\hline Valladolid & 1,18 & $1,09-1,29$ & 1,18 & $1,11-1,25$ \\
\hline Vizcaya & 1,22 & $1,15-1,30$ & 1,32 & $1,27-1,37$ \\
\hline Zamora & 1,30 & $1,13-1,51$ & 1,21 & $1,08-1,36$ \\
\hline Zaragoza & 1,18 & $1,11-1,26$ & 1,26 & $1,20-1,32$ \\
\hline Ceuta & 1,60 & $1,19-2,15$ & 1,73 & $1,45-2,07$ \\
\hline Melilla & 1,75 & $1,25-2,44$ & 1,63 & $1,27-2,08$ \\
\hline
\end{tabular}

universitarios, las tasas de mortalidad por 1.000 personas-año oscilaron entre 10,5 en Soria y 14,0 en Huelva. Otras provincias con tasas bajas y altas fueron Alicante, Orense, Cantabria, Murcia, Zamora y Tarragona, donde la magnitud fue inferior a 11 por 1.000 personas-año y Cádiz, Sevilla, Málaga, Valencia y Ciudad Real, donde la magnitud fue mayor de 13 defunciones por 1.000 personas-año.

La figuras 1 y 2 muestran, respectivamente, la distribución geográfica de la tasa de mortalidad en los sujetos con menor y mayor nivel de estudios. En los que tenían menor nivel de estudios la magnitud más alta de la tasa de mortalidad se observó en las provincias del sur y del sureste y, en el caso de los hombres, también se observó en algunas provincias del norte. Por su parte, la magnitud más baja de la tasa de mortalidad se dio en las provincias de la meseta, sobre todo en las de la submeseta norte. En cambio, en los sujetos con mayor nivel de estudios la tasa de mortalidad no mostró un patrón geográfico claro.

La razón de tasas de mortalidad en los sujetos con estudios primarios o inferiores con respecto a los que tenían estudios universitarios aparece en la tabla 3. Las razones de menor magnitud en mujeres se observaron en Palencia $(1,06)$, Segovia $(1,07)$, Guadalajara $(1,08)$, Ávila $(1,10)$ y Castellón $(1,16)$ y en hombres en Guadalajara $(1,00)$, Teruel $(1,06)$, Cuenca $(1,10)$, La Rioja $(1,12)$ y Ávila $(1,12)$. Por su parte, las estimaciones de mayor magnitud en mujeres se observaron en Melilla, Toledo, Ceuta, Las Palmas y Málaga, cuyas razones fueron $1,75,1,63,1,60,1,54$ y 1,53 , respectivamente, y en hombres las estimaciones de mayor magnitud se dieron en en Ceuta, Melilla, Murcia, Cantabria y Las Palmas, cuyas razones son $1,73,1,63,1,58,1,48$ y 1,47 , respectivamente.

En lo referente a la correlación entre las tasas de mortalidad y la renta per cápita observamos que solo se muestra una co- 
rrelación negativa estadísticamente significativa en el grupo de mujeres con estudios primarios o inferiores. Concretamente, en mujeres, los coeficientes de correlación de la renta per cápita de 2001 con la tasa de mortalidad fueron $-0,32(\mathrm{p}=0,025)$ en estudios primarios o inferiores, $-0,14(p=0,340)$ en el primer ciclo de educación secundaria, $-0,04(p=0,760)$, en segundo ciclo de educación secundaria y $-0,19 \quad(\mathrm{p}=0,185)$ en estudios universitarios. En hombres, dichos coeficientes fueron $-0.05(p=0,710)$, $-0.01(p=0,930),-0,11 \quad(p=0.430)$ y -0.23 $(\mathrm{p}=0,110)$,respectiva mente

\section{DISCUSIÓN}

La tasa de mortalidad en la población española muestra un gradiente inverso en función del nivel educativo en la práctica totalidad de las provincias. Sin embargo, el patrón geográfico de las tasas de mortalidad varía según dicho nivel educativo. Mientras que en los sujetos con menor nivel de estudios la magnitud más baja se observa en las provincias de la meseta y la magnitud más alta en las provincias del sureste, el patrón geográfico de la tasa de mortalidad en los sujetos con mayor nivel de estudios es heterogéneo. La razón de tasas de mortalidad en los sujetos con menor nivel de estudios con respecto aquellos con mayor nivel también muestra la magnitud más baja en las provincias del centro de la península.

Los atlas de mortalidad más recientes muestran mayores tasas de mortalidad en áreas de Andalucía, Murcia, Valencia, Canarias o Galicia ${ }^{1-3}$. Otras investigaciones han observado igualmente que los residentes en las áreas del sur presentan mayores tasas de mortalidad ${ }^{12-13}$. En nuestro estudio, este patrón geográfico de mortalidad se observa en los sujetos con menor nivel de estudios. Los autores de estas investigaciones han sugerido que la alta mortalidad las provincias del sur se debe a que presentan menor renta per cápita. Sin embargo, este indicador solo muestra relación con las tasas de mortalidad provincial en las mujeres con menor nivel de estudios, pero no en los otros tres grupos de nivel de estudios en mujeres ni en los cuatro grupos de nivel de estudios en hombres. Probablemente la ausencia de relación se debe a que algunas de las provincias del centro peninsular tienen una renta per cápita baja y, al mismo tiempo, también muestran tasas bajas de mortalidad.

Uno de los principales hallazgos es el hecho de que las tasas de mortalidad en los sujetos con menor nivel de estudios muestran la menor magnitud en la región central de la península, en especial en la submesesta norte. Una característica propia de las provincias de la región central de la península es que presentan un mayor porcentaje de población residente en el medio rural ${ }^{14}$. Esto podría actuar como factor protector en los individuos de nivel de estudios bajo. Existe evidencia de que las personas que viven en el medio rural presentan menor mortalidad que las que residen en el medio urbano ${ }^{15}$. Por el contrario, las ciudades tienden a concentrar las áreas de privación, lo que puede propiciar la marginalización de personas de nivel de estudios más bajo y, por tanto, aumentar las desigualdades en mortalidad en las provincias donde predomine la población residente en ciudades.

Otro hallazgo relevante es la ausencia de un claro patrón geográfico en los sujetos con nivel de estudios alto. Probablemente se deba a que las tasas de mortalidad en los sujetos con nivel de estudios alto presentan menor variabilidad geográfica que las tasas en los sujetos con nivel de estudios bajo. Por ejemplo, si se estima el coeficiente de variación en las tasas de mortalidad provinciales, se observa que su valor en las mujeres y los hombres con estudios primarios o inferiores es $12,6 \%$ y $10,1 \%$, respectivamente, mientras que en las mujeres y los hombres con estudios universitarios es $9,6 \%$ y $7,1 \%$, respectivamente. Estos resultados apoyan los hallazgos de algunas investigaciones en las que se ha encontrado que las personas con mayor nivel de estudios presentan menor 
variación en la esperanza de vida de unos lugares a otros, además de mayor longevidad. Probablemente, los sujetos con mayor educación son más capaces de acceder a una amplia variedad de recursos materiales y no materiales que les permitan optimizar su estado de salud y, como consecuencia, alargar su vida hasta una edad superior a la de las personas con menor educación ${ }^{16}$. Es posible que en las personas con nivel de estudios bajo la reducción de la mortalidad esté más relacionada con las circunstancias del contexto del área de residencia.

El hecho de que la variación geográfica en las tasas de mortalidad sea menor en los sujetos con mayor nivel de estudios condiciona que el patrón geográfico en la razón de tasas de mortalidad sea similar al patrón observado en los sujetos con menor nivel de estudios. De hecho, las razones de tasas de mortalidad muestran la mayor magnitud en las provincias del sur y la menor magnitud en las provincias del centro peninsular.

Hasta ahora sólo se disponía de estimaciones sobre desigualdades en mortalidad según el nivel de estudios en Madrid, País Vasco y Barcelona $^{17,18}$. Si se ordenan jerárquicamente las razones de tasas de mortalidad obtenidas en este trabajo en función de su magnitud, se observa que Barcelona se encuentra en el tertil de las provincias con la razón de tasas más alta en mujeres y en hombres. Por su parte, las provincias del País Vasco y Madrid se encuentran en el tertil de provincias con la razón de tasas más baja en mujeres y en el tertil de provincias con la razón de tasas intermedia en hombres.

Los hallazgos del presente trabajo difieren de los derivados del estudio MEDEA ${ }^{7,8}$. En el caso de las mujeres, el presente trabajo muestra mayores desigualdades en mortalidad en las provincias de Bilbao, Castellón, Córdoba y Pontevedra que en las ciudades de esas mismas provincias según el estudio MEDEA, mientras que en Valencia y en Málaga sucede lo contrario. En el caso de los hombres, el presente trabajo presenta mayores desigualdades en mortalidad en las provincias de Pontevedra y Córdoba con respecto a las observadas en las ciudades de esas provincias en el estudio MEDEA y lo contrario ocurre en Alicante, Bilbao, Madrid y Sevilla. Probablemente, esa discrepancia se deba a que en el estudio MEDEA los sujetos estaban clasificados en función de un indicador de privación socioeconómica del área de residencia y se ciñen a las áreas metropolitanas. Además, es posible que parte de las diferencias de los resultados en ciudades y a nivel provincial se deban al hecho de que las grandes metrópolis tienden a presentar mayores desigualdades al concentrar áreas de privación socioeconómica ${ }^{7}$.

El presente trabajo no presenta problemas de validez externa dado que representa a toda la población del territorio español durante el periodo de estudio. Así mismo, es la primera vez que se presentan tanto el patrón geográfico de mortalidad según el nivel de estudios como el patrón geográfico de las desigualdades en mortalidad.

El uso del nivel educativo como indicador de nivel socioeconómico presenta una buena comparabilidad y es capaz de clasificar a toda la población y no solo a la activa laboralmente. Sin embargo, no se ha considerado utilizar otros indicadores de posición socioeconómica, tales como nivel de ingresos, porque no estaba recogido en el censo o la ocupación dado que solo se disponía de información de los sujetos con un empleo en la semana anterior a la fecha de la realización del censo.

En conclusión, el patrón geográfico de las tasas de mortalidad en España varía según el nivel educativo. Mientras que en los sujetos con menor nivel de estudios la tasa más baja se observa en las provincias de la meseta y la más alta en las provincias del sureste, el patrón geográfico de la tasa de mortalidad en los sujetos con mayor nivel de estudios es heterogéneo. La razón de tasas de mortalidad entre los sujetos con menor y mayor nivel educativo también muestra la magnitud más baja en las provincias del centro de la península. 


\section{BIBLIOGRAFÍA}

1. Benach J(Dir.). Estudio geográfico de la mortalidad en España Análisis de tendencias temporales en municipios o agregados de municipios. Madrid: Fundación BBVA; 2007.

2. Benach J, Yasui Y, Borrell C, Rosa E, Pasarín MI, Benach $\mathrm{N}$, et al. Examining geographic patterns of mortality. The Atlas of mortality in small areas in Spain (1987-1999). Eur J Public Health1.2003; 11-123.

3. Borrell C, Cano-Serral G, Martínez-Beneito MA, Marí-Dell'Olmo M, Maica Rodríguez-Sanz, y el grupo MEDEA. Atlas de mortalidad en ciudades de España (1996-2003). Barcelona: Dit I Fet; 2009. Disponible en: http://www.aspb.es/quefem/docs/libro_atlas_alta_2009_inter.pdf

4. Borrell C, Regidor E, Arias LC, Navarro P, Puigpinós $\mathrm{R}$, Domínguez V, et al. Inequalities in mortality according to educational level in two large Southern European cities. Int J Epidemiol. 1999;28:58-63.

5. Mackenbach JP, Stirbu I, Roskam A-JR, Schaap MM, Menvielle G, Leinsalu M, et al. Socioeconomic inequalities in health in 22 European countries. N Engl J Med. 2008;358:2468-81

6. Huisman M, Kunst AE, Bopp M, et al. Educational inequalities in cause-specific mortality in middle-aged and older men and women in eight western European populations. Lancet 2005;365:493-500.

7. Borrell C, Marí-Dell'Olmo M, Serral G, MartínezBeneito M, Gotsens M, other MEDEA members. Inequalities in mortality in small areas of eleven Spanish cities (the multicenter MEDEA project). Health Place. 2010;16:703-11.

8. Martinez-Beneito MA, Zurriaga O, Botella-Rocamora et al. Do socioeconomic inequalities in mortality vary between different Spanish cities? a pooled cross-sectional analysis. BMC Public Health. 2013 May 16;13:480.

9. Jenks, George F. The Data Model Concept in Statistical Mapping. International Yearbook of Cartography. 1967;7:186-190.

10. Coulson MRC. In The Matter Of Class Intervals For Choropleth Maps: With Particular Reference To The Work Of George F Jenks Cartographica. 1987; 24 (2): 16-39.

11. Greenland S, Rothman KJ. Introduction to stratified analysis. In: Rothman KJ, Greenland S (eds). Modern Epidemiology. Philadelphia: Lippincott Williamsn \& Wilkins;1998.p.253-280.

12. Benach J, Yasui Y, Martínez JM, Borrell C, Pasarín M, Daponte A. The geography of the highest mortality areas in Spain: a striking cluster in the southwestern region of the country. Occup Environ Med 2004; 61: 280-281.
13. Benach J, Yasui Y. Geographical patterns of excess mortality in Spain explained by two indices of deprivation. J Epidemiol Community Health1999;53:423-431

14. Instituto Nacional de Estadística. Censo de Población y Viviendas. Madrid: INE; 2001. (citado el 2 de diciembre de 2014). Disponible en: http://www.ine.es/ en/inebmenu/mnu_cifraspob_en.htm

15. Ocaña-Riola, Sánchez-Cantalejo C, FernándezAjuria A. Rural habitat and risk of death in small areas of Southern Spain. Soc Sci Med 2006; 2006; 63: 13521362 .

16. Ross CE, Wu CL. Education, age, and the cumulative advantage in health. J Health Soc Behav. 1996; 37: 104-20.

17. Huisman M, Kunst AE, Bopp M, Borgan J-K, Borrell C, Costa G, et al. Educational inequalities in cause-specific mortality in middle-aged and older men and women in eight western European populations. Lancet. 2005 Feb 5;365(9458):493-500.

18. Mackenbach JP, Stirbu I, Roskam A-JR, Schaap MM, Menvielle G, Leinsalu M, et al. Socioeconomic inequalities in health in 22 European countries. N Engl J Med. 2008 Jun 5;358(23):2468-81. 